\title{
Value of serial stereotactic biopsies and impedance monitoring in the treatment of deep brain tumours
}

\author{
G I O V A N I B R OGG I A D A NGELO FRANZINI \\ From the Department of Neurosurgery, Istituto Neurologico "C Besta," Milan, Italy
}

SUMMARY Thirty-five patients with deep brain tumours have been submitted to transtumoral stereotactic impedance monitoring and serial biopsy. The direct examination of the biopsy samples confirmed the presumptive clinical and neuroradiological diagnosis in 25 patients, but in 10 patients the histological diagnosis differed from the presumptive one. In this second group the treatment was changed as a result of the histological findings. Stereotactic biopsy avoided the risks of "blind" management. The technique, the indications and the diagnostic advantages of stereotactic biopsy are reported with two illustrative cases.

The surgical treatment of deep brain tumours may be by microsurgery, ${ }^{1}$ stereotactic irradiation, ${ }^{2-4}$ stereotactic cryosurgery, ${ }^{5}$ or stereotactic radiof requency coagulations. ${ }^{6}$ Chemotherapy and radiotherapy are used for non-invasive treatment in selected cases. ${ }^{8}$ The choice between different treatments is easier if the histological nature of the neoplasm is known. ${ }^{-11}$

A reliable method of learning the histological nature of a deep brain tumour is by serial stereotactic biopsy using the CT scan and guided by transtumoral impedance monitoring. ${ }^{12}{ }^{13}$ In this paper we discuss the indications, the diagnostic advantages and the technique of this procedure.

\section{Material and methods}

Of 306 patients with brain tumours, admitted to our department in 1979,35 patients with deep seated lesions underwent stereotactic biopsy and impedance monitoring of the tumour and adjacent tissues. In each patient the tumour was first localised by angiography and $C T$. In nine patients the mass was in the basal ganglia region, in seven patients in the frontal subcortical white matter, in five patients in the temporal subcortical white matter, in two patients in the parietal subcortical white matter, in two patients in the pineal region, in three patients in the midbrain, and in seven patients in the sellar and suprasellar regions (table).

Address for reprint requests: Dr Giovanni Broggi, Istituto Neurologico "C Besta", Via Celoria, 11, 20133 Milano, Italy.

Accepted 21 January 1981
The tomodensitometric image of the tumour was transposed to stereotactic plain radiographs following a graphic and mathematical method similar to the one described by Gleason et al. ${ }^{6}$ Multiple targets within the mass were chosen in order to obtain several tissue samples from different areas of the tumour; the targets were along linear penetrating trajectories passing through the tumour from the superficial to the deep boundaries.

When the CT scan showed heterogeneous densities in the tumour, the targets were calculated to investigate the focal abnormalities within the neoplasm by several trajectories.

In 30 cases the stereotactic co-ordinates of a single linear trajectory were calculated allowing five to eight targets, while in five patients more trajectories were used. The Riechert stereotactic apparatus was employed and the whole procedure was carried out under general anaesthesia. Stereotactic ventriculography with positive contrast medium (Dimer $\mathbf{X}$, or Amipaque, or Conray), was used in the procedure.

A $9 \mathrm{~mm}$ diameter burr hole was made in the frontal bone and a stainless steel electrode (diameter $0.8 \mathrm{~mm}$ ) was placed within a cannula (diameter $1.5 \mathrm{~mm}$ ) and inserted and guided along the chosen trajectory by the stereotactic apparatus; the electrode had a bare tip protruding from the cannula (length $2 \mathrm{~mm}$ ) recording the impedance values and connected to the impedance recorder (Radionic apparatus RFG 5s working at $50 \mathrm{kHz}, 100 \mu \mathrm{A}$ current). The detected impedance profile was correlated with the depth of the electrode and with the stereotactic co-ordinates of the mass in order to obtain the impedance value of the tumour and adjacent tissues ${ }^{13}$ (figs 1 and 2) confirming the real boundaries of the neoplasm. 
Table Summary of whole series. Cases in which the serial stereotactic biopsy changed the previous clinical and neuroradiological diagnosis and allowed a more correct therapeutical management. $\square$ Cases in which the clinical and neuroradiological diagnosis was confirmed by the serial stereotactic biopsy

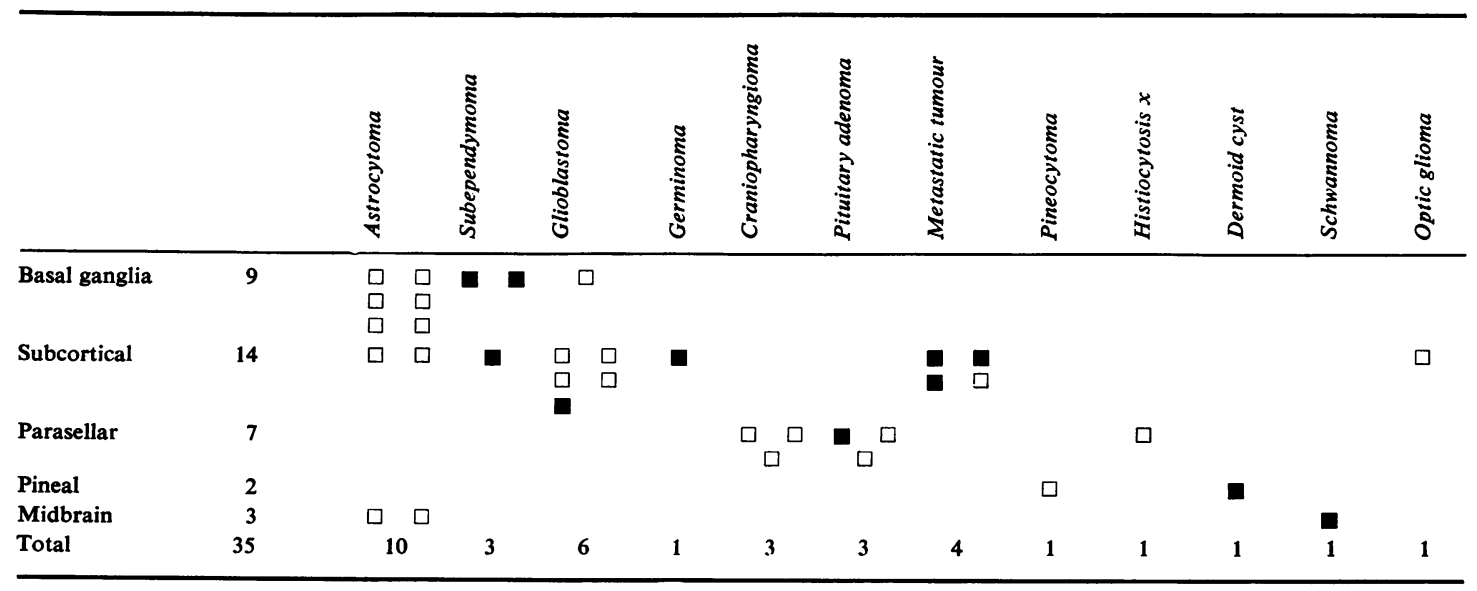

The electrode (diameter $0.9 \mathrm{~mm}$, Fischer Co Freiburg) was then removed and biopsy forceps introduced along the same trajectory. The tissue samples were taken out, at the chosen target, using both CT transposed data and the impedance profile. Part of each sample was examined at once by smear technique and methylene blue staining 1415 to confirm the

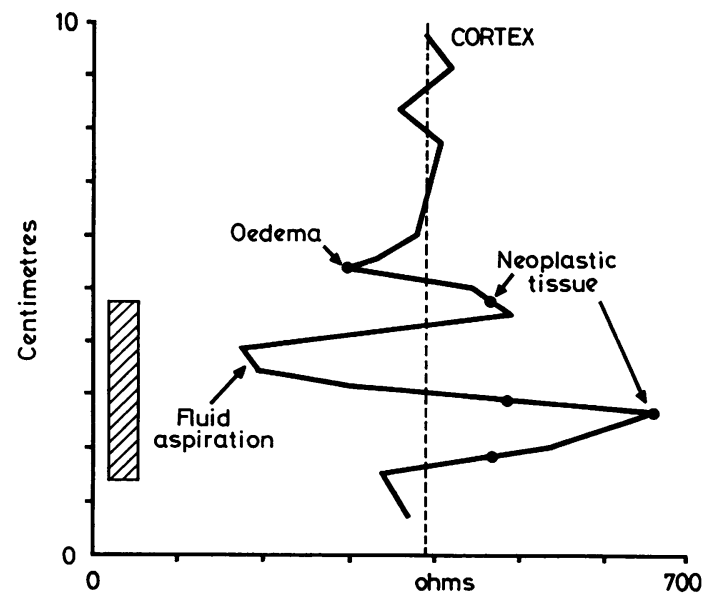

Fig 1 Impedance profile of case 1. Ordinate: depth from the cortex to the target along the penetration line. Abscissa: impedance values. The dotted line outlines the impedance value recorded in the cortex. The hatched area on the left represents the estimated stereotactic co-ordinates of the tumour derived from CT scan and angiographic data. Biopsy points are indicated by black spots. Note the different impedance values corresponding to serial biopsy specimens and the lower value in the cystic area. presence of neoplastic tissue; a major part of the sample was embedded in paraffin for histological examination.

\section{Results}

The site and histological diagnoses are given in the table.

The presumptive diagnosis based on clinical and neuroradiological data was confirmed by direct histological examination in 25 patients of this series, while in 10 cases the histological diagnosis was different from the presumptive one. In this second group the planned therapy was changed

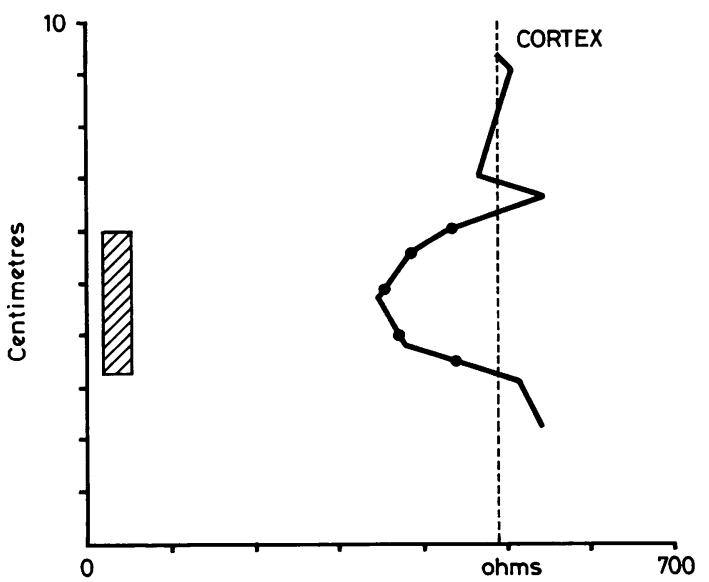

Fig 2 Impedance profile of case 2. Ordinate and abscissa as in figure 1. Note that neoplastic tissue has an impedance value lower than cortex. 
following the histological diagnosis. Three patients in whom clinical and neuroradiological data suggested astrocytomas deep in the basal ganglia and temporal region, actually had subependymomas, and microsurgical ablation was performed instead of radiotherapy. Three patients with midline tumours suggesting intra-axial growths were assigned to radiotherapy, but the histological diagnosis showed extra-axial neoplasms: Schwannoma (of the third cranial nerve) in the midbrain region, dermoid cyst in the pineal region and pituitary adenoma in the hypothalamic supraretrosellar region: all underwent open surgery. Three patients, following the presumptive diagnosis of subcortical glioblastomas, were assigned to radiotherapy. The histological findings showed radioresistant metastatic tumours and surgical ablation was performed in two cases, while in the third patient chemotherapy was attempted. One patient whose clinical history and neuroradiology suggested a metastatic tumour deep in the parietal white matter, had an ectopic germinoma and was successfully treated by external radiotherapy. All these data are summarised in the table.

In the whole series of patients, the side-effects of stereotactic procedure were worsening of preexistent third nerve paresis in one patient affected by brain stem astrocytoma and reversible worsening of hemiparesis in two patients affected by thalamic tumours. There was no mortality in this series. Two cases from this series are reported in detail.

\section{Case 1}

A forty-five-year-old man suffered from slowly progressive right hemiparesis with mild central palsy of the facial nerve, for five years. CT scan showed a midbrain tumour involving the left basal ganglia with widening of the lateral and third ventricles. In another hospital a ventriculo-peritoneal shunt was inserted with slight improvement of the hemiparesis. A few months later he was admitted to our hospital because
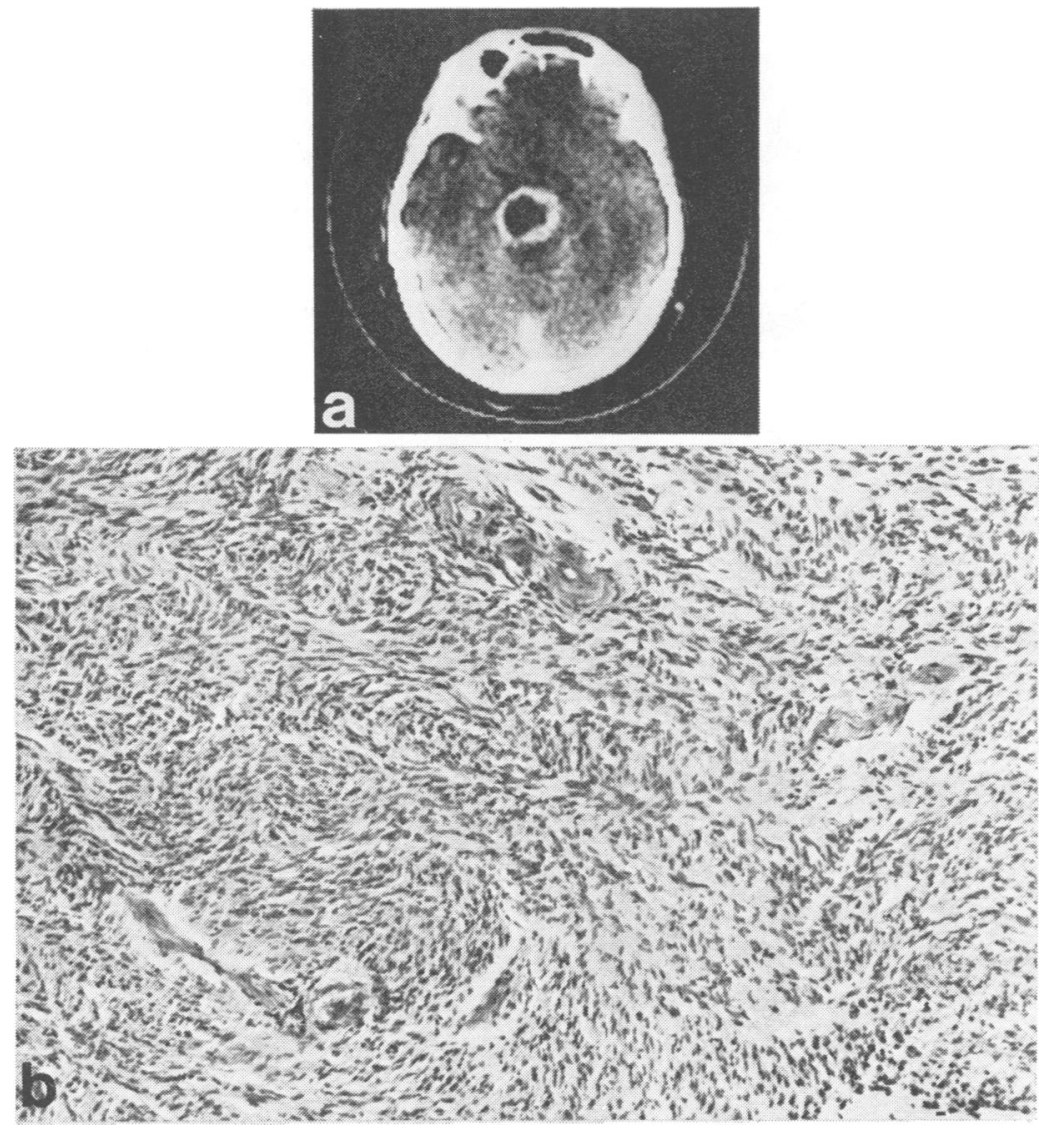

Fig 3 (a) CT scan with intravenous contrast medium of case 1. Cystic lesion with the hyperdense rim in the left basal ganglia region. (b) Histological finding in case 1 from biopsy specimen schwannoma. $H E$ stain $\times 132$ 
of onset of diplopia and worsening of the hemiparesis. CT scan examination with intravenous contrast medium showed the tumour to have a hyperdense rim surrounding a low density central area (fig 3a). Cerebral angiography showed only displacement of vessels without abnormal vessels.

The presumptive clinical and neuroradiological diagnosis was of a cystic astrocytoma of the brain stem. Serial stereotactic biopsy was performed. The impedance profile derived during the procedure showed values higher than that of the cortex in the area corresponding to the hyperdense rim, with values lower than that of the cortex at the levels corresponding to the hypodense central area of the tumour (fig 1). Tissue samples were taken from the high-impedance area (rim area in CT scan) while from the lowimpedance area only a few millilitres of yellow proteinaceous fluid could be aspired through the cannula. Histological examination of the tissue showed that the tumour was a Schwannoma (fig 3b). Fifteen days later the tumour was removed by a subtemporal approach with microsurgery and its origin from the left third nerve was discovered.

\section{Case 2}

This fifty-six-year-old man had suffered from mild right hemiparesis for three months. CT examination with intravenous contrast medium showed a hyperdense lesion involving the left basal ganglia and surrounded by a widespread hypodense area involving the parietal subcortical white matter (fig 4a). Right carotid angiography showed only displacement of the main vessels without abnormal vessels, leading to the suspicion of a metastatic tumour. Serial stereotactic biopsy and trans-tumoral impedance monitoring was performed; multiple tissue samples were taken from the low-impedance area corresponding to the tumour
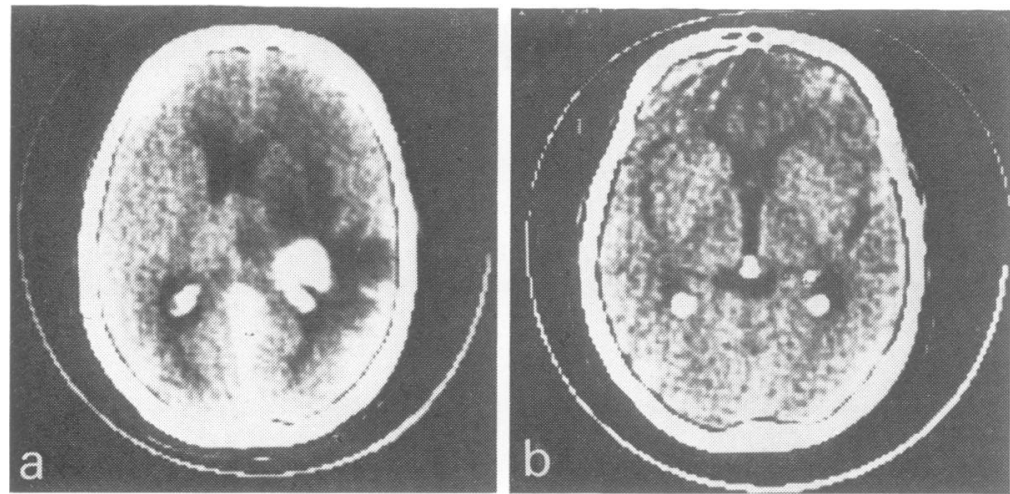

Fig 4 (a) CT scan with contrast medium enhancement of case 2 before stereotactic biopsy. Note the deep seated lesion and surrounding oedema in right parietal lobe. (b) $C T$ scan after radiation therapy (5000 rads). Note the almost complete disappearance of the tumour. Note also the metal piece stereotactically positioned at the point where the biopsy specimen of fig 5 was taken.

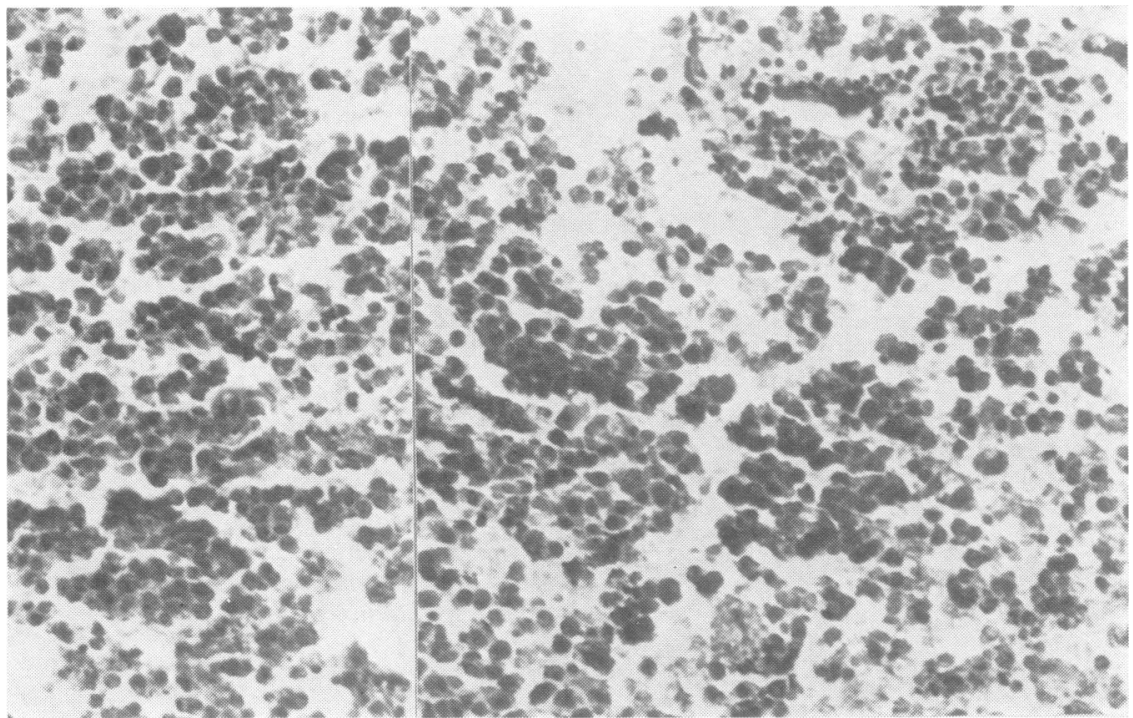

Fig 5 Histological appearance of a biopsy specimen of case 2: germinoma. HE stain $\times 485$ 
stereotactic co-ordinates (fig 2). Histological examination showed that the tumour was a germinoma (fig 5). The patient was treated by external radiotherapy with 5000 rads delivered over a period of two months. Four months later CT examination with intravenous contrast medium showed disappearance of the tumour and surrounding oedema (fig $4 \mathrm{~b}$ ). A few days after treatment began the right hemiparesis dramatically improved and one year later only slight hyperreflexia on the right side was present.

\section{Discussion}

The following conclusions are suggested.

The technique of serial stereotactic biopsy has a low risk.

Impedance monitoring may be crucial in the choice of targets, allowing the detection of different structures in the neoplasma, for example, cyst, necrotic areas, oedema and neoplastic solid tissue. Moreover real boundaries of the tumour may be ascertained.

Intraoperative imprint cytology is a useful tool to confirm the value of the targets and allows an immediate correlation with the impedance data.

The best management of deep brain tumours is assisted by a knowledge of the histological nature of the lesion, avoiding inappropriate treatment based only on clinical and neuroradiological data (inappropriate treatment was avoided in 10 cases in our series).

Finally, we conclude that nowadays no "blind" surgical or radiation treatment is justified in deep seated brain tumours in which the diagnosis is not completely certain.

This study was partially supported by the Harry de Jur Foundation and it was presented in part to the Annual congress of the Italian Society of Neurosurgery, Rome, November 1979.

\section{References}

1 Habler W, Seeger W. Microsurgery of tumours of the frontal white matter. Acta Neurochir 1978; 44:11-48.

2 Kelly PJ, Olson MH, Wright AE. Stereotactic implantation of iridium ${ }^{192}$ into CNS neoplasms. Surg Neurol 1978; 10:349-54.

3 Mundinger F, Hoefer T. Protracted long-term irradiation of inoperable midbrain tumors by stereotactic Curie-therapy using Iridium ${ }^{192}$. Acta Neurochir 1974; Suppl 21:93-100.

4 Szikla G, Schlienger M, Betti O et al. Combined interstitial and external irradiation of gliomas. In: Szikla G ed. Stereotactic Cerebral Irradiation. Amsterdam: Elsevier-North Holland Biomedical Press, 1979:329-38.

5 Conway LW. Stereotactic diagnosis and treatment of intracranial tumors including an initial experience with cryosurgery for pinealomas. $J$ Neurosurg 1973; 38:453-60.

6 Gleason CA, Burton LW, Feinstein B. Stereotactic localization (with computerized tomographic scanning), biopsy, and radiofrequency treatment of deep brain lesions. Neurosurgery 1978; 3: 217-22.

7 Walker MD, Hilton J. Nitrosurea pharmacodynamics in relation to the central nervous system. Cancer Treat Rep 1976; 60:725-37.

8 Wara WM, Sheline GE. Radiation therapy of malignant brain tumors. Clin Neurosurg 1978; 25:397-402.

9 Boethius J, Collins VP, Edner G, Lewander R, Zajicek J. Stereotactic biopsies and computer tomography in gliomas. Acta Neurochir 1978; 40: 223-32.

10 Pecker J, Scarabin JM, Vallée B. Stereotactic approach to diagnosis and treatment of cerebral tumors (Démarche stéréotaxique en neurochirurgie tumorale). Paris: Editions Fabre, 1979.

11 Waltregny A, Petrov V, Brotchi J. Serial stereotactic biopsies. Acta Neurochir 1974; Suppl 21: 221-6.

12 Benabid AL, Persat JC, Chiroussel, J et al. Correlative study between computerized transverse scanning and stereoimpedoencephalography in space-occuping lesions of the brain. Acta Neurochir 1979; 46:219-32.

13 Broggi G, Franzini A, Passerini A. Correlation between impedance values and deep brain tumors during stereotactic biopsies. In: Szikla $G$ ed. Stereotactic Cerebral Irradiation. Amsterdam: Elsevier-North Holland Biomedical Press, 1979: 51-6.

14 Eisenhardt L, Cushing H. Diagnosis of intracranial tumors by supravital technique. $A m \mathrm{~J}$ Path 1930; 6:541-52.

15 Mennel HD, Ostertag C. Stereotactic intraoperative cytological diagnosis of brain tumors. In: Szikla G ed. Stereotactic Cerebral Irradiation. Amsterdam, Elsevier-North Holland Biomedical Press, 1979; 43-9. 